\title{
Assessment of genetic diversity in the USDA and CIP-FAO international nursery collections of quinoa (Chenopodium quinoa Willd.) using microsatellite markers
}

\author{
S. A. Christensen \\ D. B. Pratt \\ C. Pratt \\ P. T. Nelson \\ M. R. Stevens
}

See next page for additional authors

Follow this and additional works at: https://scholarsarchive.byu.edu/facpub

Part of the Animal Sciences Commons, and the Plant Sciences Commons

\section{Original Publication Citation}

Plant Genetic Resources 5.2(Aug 27): 82-95.

\section{BYU ScholarsArchive Citation}

Christensen, S. A.; Pratt, D. B.; Pratt, C.; Nelson, P. T.; Stevens, M. R.; Jellen, Eric N.; Coleman, Craig E.; Fairbanks, Daniel J.; Bonifacio, A.; and Maughan, Michael J., "Assessment of genetic diversity in the USDA and CIP-FAO international nursery collections of quinoa (Chenopodium quinoa Willd.) using microsatellite markers" (2007). Faculty Publications. 242.

https://scholarsarchive.byu.edu/facpub/242

This Peer-Reviewed Article is brought to you for free and open access by BYU ScholarsArchive. It has been accepted for inclusion in Faculty Publications by an authorized administrator of BYU ScholarsArchive. For more information, please contact ellen_amatangelo@byu.edu. 


\section{Authors}

S. A. Christensen, D. B. Pratt, C. Pratt, P. T. Nelson, M. R. Stevens, Eric N. Jellen, Craig E. Coleman, Daniel J. Fairbanks, A. Bonifacio, and Michael J. Maughan 


\title{
Assessment of genetic diversity in the USDA and CIP-FAO international nursery collections of quinoa (Chenopodium quinoa Willd.) using microsatellite markers
}

\author{
S. A. Christensen ${ }^{1}$, D. B. Pratt ${ }^{2}$, C. Pratt ${ }^{1}$, P. T. Nelson ${ }^{1}$, M. R. Stevens ${ }^{1}$, \\ E. N. Jellen ${ }^{1}$, C. E. Coleman ${ }^{1}$, D. J. Fairbanks ${ }^{1}$, A. Bonifacio ${ }^{3}$ and \\ P. J. Maughan ${ }^{1 *}$ \\ ${ }^{1}$ Brigham Young University, Department of Plant \& Animal Sciences, 275 WIDB, Provo, \\ UT 84602, USA, ${ }^{2}$ Stephen F. Austin State University, Department of Biology, Nacogdoches, \\ TX 75961, USA and ${ }^{3}$ Fundacion PROINPA, Casilla Postal 4285, Cochabamba, Bolivia
}

Received 19 January 2006; Accepted 18 September 2006

\begin{abstract}
Quinoa (Chenopodium quinoa Willd.) is a staple food crop for millions of impoverished rural inhabitants of Andean South America where it has been cultivated for millennia. Interest in quinoa, due largely to its superior nutritional characteristics, is fuelling a growing export market and has led to an increased focus on genetic research and the development of quinoa breeding programmes throughout South America. The success of these breeding programmes will rely heavily on the development of core germplasm collections and germplasm conservation. We report the development of a set of fluorescence-tagged microsatellite molecular markers that can be used to characterize genetic diversity within quinoa germplasm and we use this set of 36 microsatellites markers to genetically characterize the diversity of 121 accessions of C. quinoa held in the USDA germplasm bank, 22 accessions from the CIP-FAO international nursery collection and eight accessions representing parents from genetic mapping populations. A total of 420 alleles were detected among the quinoa accessions with an average of 11 alleles detected per microsatellite locus. Genetic heterogeneity was observed in $32 \%$ of the quinoa accessions at a given locus and suggests that many of these accessions represent heterogeneous seed lots or landraces. Both unweighted pairgroup method with arithmetic averages (UPGMA) and principle components analysis (PCA) analyses partitioned the quinoa accessions into two main clusters. The first major cluster consisted of accessions from the Andean highlands of Peru, Bolivia, Ecuador, Argentina and extreme northeastern Chile. The other main cluster contained accessions from both the lowlands of Chile and a set of USDA accessions with no known passport data, collected by Emigdio Ballón. Using the patterns of genetic diversity detected within the C. quinoa accessions we discuss hypotheses regarding quinoa's centre of diversity, including highland and lowland ecotype clustering patterns, origin of lowland varieties, origin of domestication, and diversity levels in the USDA and CIP-FAO collections.
\end{abstract}

Keywords: Chenopodium quinoa; genetic diversity; microsatellites; SSRs

\section{Introduction}

*Corresponding author. E-mail: jeff_maughan@byu.edu

Quinoa (Chenopodium quinoa Willd.) is one of the most important food crops of the Andean region of South America (Cusack, 1984). It is an allotetraploid 
$(2 n=4 x=36)$ that shows disomic inheritance for most qualitative traits (Simmonds, 1971; Risi and Galwey, 1984; Ward, 2000). The seeds of quinoa are high in protein $(\sim 16 \%)$ and contain an excellent balance of the amino acids essential to the human diet (Ruales and Nair, 1992; Ruales et al., 2002). Quinoa is uniquely adapted to the harsh conditions that characterize much of the Andean Altiplano (high plains), including high altitudes $(3500-3850 \mathrm{~m}$ asl), arid $(80-600 \mathrm{~mm} /$ year) and saline soils as well as frequent frost.

Heightened awareness of quinoa's role in food security issues in Andean South America, and its unique potential as a crop for marginal soils worldwide, and a growing health food export market for quinoa, has led to increased interest in quinoa and the establishment of several new breeding programmes throughout South America. Essential to these breeding programmes is the development of quinoa germplasm collections. We estimate that there are approximately 5000 diverse landraces of quinoa maintained in collections worldwide, including 2700 in Bolivia, 1029 in Peru (Ortiz et al., 1998) and 232 in the USDA National Plant Germplasm System (NPGS). An undocumented number of accessions exist in Chile, Argentina, Ecuador and Colombia (Jacobsen, 2000; Rojas et al., 2000; F. Fuentes, personal communication).

There are relatively few published efforts to characterize genetic diversity within quinoa germplasm collections. Wilson (1988b) used morphological data combined with data from six isozyme loci to characterize 98 diverse quinoa accessions. His research suggested that (i) the centre of quinoa diversity is likely located in the southern Andean highlands; (ii) accessions of quinoa cluster into two main groups: Andean highland and coastal lowland (hereafter denoted as highland and lowland); (iii) the lowland varieties originated from the southern highland populations; and (iv) the origin of domestication was likely located in the Peruvian/Bolivian region with subsequent dispersals to the north and south.

Ortiz et al. (1988) characterized the 1029 accessions of the Peruvian quinoa germplasm bank at the Universidad National del Altiplano-Puno (UNAP) using eight phenotypic descriptors. Their goal was to derive a representative core collection of 103 accessions using a sampling strategy based primarily on altitude of origin in a geographically stratified, non-redundant procedure. They reported that quantitative trait variation and altitude were highly correlated and demostrated the ability to represent a majority of the range of variation with a $10 \%$ core subset.

In another study, Rojas et al. (2000) analysed phenotypic variation within 1512 accessions of the Bolivian national quinoa collection using three multivariate procedures for 17 traits (15 quantitative, two qualitative). Their study included genotypes collected in Chile, Argentina and
Peru, as well as native Bolivian materials. Using principal component, cluster and discriminate function analyses, they classified quinoa germplasm into seven distinct groups, including five within the Altiplano and two from lower altitude valleys of the eastern Andean mountain range. Their characters did not discriminate for the Chilean lowland germplasm, which grouped with one of their Altiplano clusters.

The ability to identify genetic variation is indispensable to effective germplasm management and conservation (Roa, 2004). Molecular markers provide unique and effective tools for evaluating and characterizing plant genetic diversity in a way that is unaffected by the environment (Gupta and Varshney, 2000). Indeed, molecular marker information has proven valuable in several aspects of germplasm management, including (i) identification of genetic identities within collections, (ii) development of genetically diverse core collections (a genetically representative subset of the reserve collection), (iii) monitoring of natural and artificial changes in the genetic collections (e.g. contaminants, hybridizations or mixtures) and (iv) identification of phylogenetic relationships within the germplasm collection and with related weedy species (Hokanson et al., 1998; Dean et al., 1999; McGregor et al., 2002). Among the various molecular markers, microsatellites (also known as simple sequence repeats or SSRs), have emerged as the genetic marker system of choice for plant genetic resource analysis, owing to their ease of use, highly polymorphic nature and abundance throughout plant genomes (Maughan et al., 1996; Mace and Godwin, 2002).

The conclusions derived from Wilson (1988a, b) and the development of the first set of quinoa microsatellite markers (Mason et al., 2005) provide a foundation for an in-depth molecular survey of genetic diversity in this species. We report on the use of 36 fluorescencetagged SSRs to genetically characterize 153 quinoa accessions from the USDA-NPGS and CIP-FAO collections. Using the patterns of genetic diversity detected within the C. quinoa accessions we discuss Wilson's (1988a, b) hypotheses regarding quinoa's centre of diversity, including highland and lowland clustering patterns, origin of lowland varieties and the origin of domestication.

\section{Materials and methods}

\section{Plant material and DNA isolation}

The C. quinoa plant material utilized in this investigation were derived from several sources, namely 121 accessions from the USDA North Central Regional Plant Introduction 
Station of the US National Plant Germplasm System (Ames, IA; David Brenner), 22 accessions that make up the publicly available international nursery collection developed by the Centro Internacional de la Papa (CIP; Mujica et al., 1998; Angel Mujica, National University of the Altiplano, Puno, Peru) and eight accessions used as parents in several genetic mapping populations provided by Alejandro Bonifacio (The Foundation for the Promotion and Investigation of Andean Products; PROINPA) (Table 1). We note that the USDA Chenopodium collection consists of 41 accessions included in this analysis that were collected by Emigdio Ballón (Traditional Native American Farmers Association, NM, USA) for which passport (origin) data are unavailable. Hereafter we refer to this specific portion of the USDA collection as the Ballón collection. Personal conversations with Emigdio Ballón suggest that this material was increased in New Mexico prior to its donation to the USDA collection on 15 May 1990.

Additionally, one accession of $C$. giganteum D. Don (Ames 19046; a related chenopod crop species) and one accession of C. album L. (PI 433378; lambsquarters or fat hen) were also provided by David Brenner (USDA-NPGS, Ames, IA) and included in our analysis for comparative purposes.

All plant material was grown at $25^{\circ} \mathrm{C}$ with a $12 \mathrm{~h}$ photoperiod in greenhouses at Brigham Young University, Provo, UT, USA. Equal amounts of leaf material were harvested from three individuals and bulked for DNA isolation using a freeze-dried leaf tissue protocol described by Sambrook et al. (1989) with modifications described by Todd and Vodkin (1996).

\section{Fluorescent SSR analysis}

In total, 36 highly reproducible microsatellite primer pairs that produced a simple banding pattern (i.e. all markers amplified a single polymorphic band with the exception of one marker (AAT71) that amplified a second monomorphic band) in prescreen tests and exhibited high heterozygosity values (range $0.33-0.85$; mean $=0.59$; Mason et al., 2005) were selected for genotyping. Thirty-two of the microsatellite loci were previously described by Mason et al. (2005), while the remaining four were developed by P. J. Maughan et al. (unpublished data). GenBank accession numbers for all loci are reported in Table 2 .

Fluorescent labels were added to either the forward or reverse primer with the strongest $3^{\prime}$ end (i.e. the end with the fewest mononucleotide repeats; Table 2). Each fluorescently labelled primer was synthesized by Applied Biosystems (Foster City, CA, USA) with one of the following fluorophores: 6-FAM, TET, HEX, VIC or NED (Table 2). Since all primer pairs for each of the microsatellite loci were originally designed to amplify PCR fragments in a narrow base pair range $(150-250 \mathrm{bp})$ to allow maximal size separation (Mason et al., 2005), allele size multiplexing was not attempted in this experiment. To avoid crossamplification among primer pairs, each microsatellite was amplified separately in uniplex PCR reactions. PCR reactions consisted of $60 \mathrm{ng}$ of quinoa genomic DNA, $0.2 \mathrm{mM}$ dNTPs, $2.5 \mathrm{mM} \mathrm{MgCl}_{2}, 1 \times$ PCR buffer, $1.0 \mu \mathrm{M}$ primer and $1 \mathrm{U}$ of Taq polymerase in a reaction volume of $15 \mu \mathrm{l}$. The amplification profile was as follows: 18 cycles of $94^{\circ} \mathrm{C}$ for $60 \mathrm{~s}, 67^{\circ} \mathrm{C}$ for $30 \mathrm{~s}, 72^{\circ} \mathrm{C}$ for $60 \mathrm{~s}$, followed by 20 cycles using $58^{\circ} \mathrm{C}$ as annealing temperature, followed by a final 10 min extension step at $72^{\circ} \mathrm{C}$. After amplification, PCR products labelled with three different fluorophores were pooled by combining $2 \mu \mathrm{l}$ aliquots from each and diluting in $64 \mu \mathrm{l}$ of HPLC-grade $\mathrm{H}_{2} \mathrm{O}$. A $2 \mu \mathrm{l}$ aliquot of the pooled dilution was then dried down and submitted for size analysis at the BYU DNA sequencing centre (Provo, UT, USA). A size standard (GS-500) containing DNA fragments ranging in size from 35 to $500 \mathrm{bp}$ labelled with the fluorescent dye ROX was added to each pooled sample. Amplified fragments were separated electrophoretically using an ABI 3100 Genetic Analyzer (Applied Biosystems).

\section{Data analysis}

Fluorescent peaks were analysed with the GENESCAN prism software version 3.7. Microsatellite alleles sizes were estimated with the GENOTYPER software version 2.1 (Applied Biosystems) and scored using the local southern base calling method for allele sizing (Elder and Southern, 1987). The microsatellite data set was converted to binary digits for presence (1) or absence (0) of specific alleles. Loci and accessions for which $25 \%$ or greater of the data were missing were removed from the data set. Various diversity measures were calculated from the resulting data, including mean number of alleles per locus, total number of alleles and number of unique alleles. Heterozygosity values for each marker locus were determined following published procedures (Mason et al., 2005).

The data were subsequently analysed using the SIMQUAL subprogram of NTSYS-PC (Rohlf, 2000) to generate similarity matrices. These similarity matrices were subsequently analysed phenetically using both clustering and ordination algorithms in NTSYS-pc (Rohlf, 2000). An unweighted pair-group method with arithmetic averages (UPGMA) dendrogram was constructed using a double-centred Jaccard's similarity matrix and rooted using C. album and C. giganteum as outgroups. Principle components analysis (PCA) was performed using a variance-covariance matrix of the data. 
Table 1. Passport data on Chenopodium accessions used for microsatellite analysis

\begin{tabular}{|c|c|c|c|}
\hline Sample ID/name & Accession no. & Passport/origin ${ }^{\mathrm{a}}$ & Source ${ }^{b}$ \\
\hline Jujuy & $\mathrm{N} / \mathrm{A}$ & Argentina, Jujuy & CIP-FAO \\
\hline Kamira & $\mathrm{N} / \mathrm{A}$ & Bolivia & CIP-FAO \\
\hline Ratuqui & $\mathrm{N} / \mathrm{A}$ & Bolivia & CIP-FAO \\
\hline Real & $\mathrm{N} / \mathrm{A}$ & Bolivia, Oruro & CIP-FAO \\
\hline 02-Embrapa & $\mathrm{N} / \mathrm{A}$ & Brazil & CIP-FAO \\
\hline Ollague & $\mathrm{N} / \mathrm{A}$ & Chile & CIP-FAO \\
\hline Narino & $\mathrm{N} / \mathrm{A}$ & Columbia & CIP-FAO \\
\hline E-DK-4 & $\mathrm{N} / \mathrm{A}$ & Denmark_Peruvian origin & CIP-FAO \\
\hline G-205-95 & $\mathrm{N} / \mathrm{A}$ & Denmark-Peruvian origin & CIP-FAO \\
\hline ECU-420 & $\mathrm{N} / \mathrm{A}$ & Ecuador & CIP-FAO \\
\hline Ingapirca & $\mathrm{N} / \mathrm{A}$ & Ecuador & CIP-FAO \\
\hline RU-2 & $\mathrm{N} / \mathrm{A}$ & England-Chilean origin & CIP-FAO \\
\hline RU -5 & $\mathrm{~N} / \mathrm{A}$ & England-Chilean origin & CIP-FAO \\
\hline NL-6 & $\mathrm{N} / \mathrm{A}$ & Holland-Chilean origin & CIP-FAO \\
\hline CICA-127 & $\mathrm{N} / \mathrm{A}$ & Peru, Cusco & CIP-FAO \\
\hline CICA-17 & $\mathrm{N} / \mathrm{A}$ & Peru, Cusco & CIP-FAO \\
\hline $03-21-072 \mathrm{RM}$ & $\mathrm{N} / \mathrm{A}$ & Peru, Puno & CIP-FAO \\
\hline $03-21-079 B B$ & $\mathrm{~N} / \mathrm{A}$ & Peru, Puno & CIP-FAO \\
\hline Huariponcho & $\mathrm{N} / \mathrm{A}$ & Peru, Puno & CIP-FAO \\
\hline Illpa & $\mathrm{N} / \mathrm{A}$ & Peru, Puno & CIP-FAO \\
\hline Kancolla & $\mathrm{N} / \mathrm{A}$ & Peru, Puno & CIP-FAO \\
\hline Salcedo & $\mathrm{N} / \mathrm{A}$ & Peru, Puno & CIP-FAO \\
\hline Maniquena & $\mathrm{N} / \mathrm{A}$ & Bolivia & PROINPA \\
\hline Mocko & $\mathrm{N} / \mathrm{A}$ & Bolivia & PROINPA \\
\hline Chucapaca & $\mathrm{N} / \mathrm{A}$ & Bolivia & PROINPA \\
\hline $\mathrm{L}-\mathrm{P}$ & $\mathrm{N} / \mathrm{A}$ & Bolivia & PROINPA \\
\hline Surumi & $\mathrm{N} / \mathrm{A}$ & Bolivia & PROINPA \\
\hline $\mathrm{KU}-2$ & $\mathrm{~N} / \mathrm{A}$ & Chile & PROINPA \\
\hline G-205-95DK & $\mathrm{N} / \mathrm{A}$ & Denmark-Chilean origin & PROINPA \\
\hline 0654 & $\mathrm{~N} / \mathrm{A}$ & Peru, Puno & PROINPA \\
\hline A1 & PI 614881 & Argentina, Jujuy & USDA-NPGS \\
\hline $\mathrm{A} 2$ & PI 614883 & Argentina, Jujuy & USDA-NPGS \\
\hline A3 & PI 614884 & Argentina, Jujuy & USDA-NPGS \\
\hline A4 & PI 587173 & Argentina, Jujuy & USDA-NPGS \\
\hline B2 & PI 614902 & Bolivia, Oruro & USDA-NPGS \\
\hline B3 & PI 614904 & Bolivia, Oruro & USDA-NPGS \\
\hline B4 & PI 614905 & Bolivia, Oruro & USDA-NPGS \\
\hline B6 & PI 614907 & Bolivia, Oruro & USDA-NPGS \\
\hline B8 & PI 614909 & Bolivia, Oruro & USDA-NPGS \\
\hline B9 & PI 614910 & Bolivia, Oruro & USDA-NPGS \\
\hline B10 & PI 614911 & Bolivia, Oruro & USDA-NPGS \\
\hline B11 & PI 614912 & Bolivia, Oruro & USDA-NPGS \\
\hline B12 & PI 614913 & Bolivia, Oruro & USDA-NPGS \\
\hline B13 & PI 614915 & Bolivia, Oruro & USDA-NPGS \\
\hline B14 & PI 614916 & Bolivia, Oruro & USDA-NPGS \\
\hline B15 & PI 614919 & Bolivia, Oruro & USDA-NPGS \\
\hline B16 & PI 614920 & Bolivia, Oruro & USDA-NPGS \\
\hline B23 & PI 614927 & Bolivia, La Paz & USDA-NPGS \\
\hline B24 & PI 614928 & Bolivia, La Paz & USDA-NPGS \\
\hline B25 & PI 614929 & Bolivia, La Paz & USDA-NPGS \\
\hline B27 & PI 614931 & Bolivia, Oruro & USDA-NPGS \\
\hline B28 & PI 614932 & Bolivia, Oruro & USDA-NPGS \\
\hline B29 & PI 614933 & Bolivia, Oruro & USDA-NPGS \\
\hline B30 & PI 614934 & Bolivia, Oruro & USDA-NPGS \\
\hline B31 & PI 614935 & Bolivia, Oruro & USDA-NPGS \\
\hline B32 & PI 614936 & Bolivia, Oruro & USDA-NPGS \\
\hline B33 & PI 614937 & Bolivia, Oruro & USDA-NPGS \\
\hline B34 & PI 614938 & Bolivia, Oruro & USDA-NPGS \\
\hline B35 & PI 478415 & Bolivia, La Paz & USDA-NPGS \\
\hline B36 & PI 478408 & Bolivia, La Paz & USDA-NPGS \\
\hline
\end{tabular}


Table 1. Continued

\begin{tabular}{|c|c|c|c|}
\hline Sample ID/name & Accession no. & Passport/origin $^{a}$ & Source $^{b}$ \\
\hline B36 & PI 478418 & Bolivia, Potosi & USDA-NPGS \\
\hline B37 & PI 478410 & Bolivia, La Paz & USDA-NPGS \\
\hline B38 & PI 478414 & Bolivia, La Paz & USDA-NPGS \\
\hline B39 & PI 614002 & Bolivia, Cochabamba & USDA-NPGS \\
\hline B40 & Ames 13215 & Bolivia, La Paz & USDA-NPGS \\
\hline B42 & Ames 13217 & Bolivia, La Paz & USDA-NPGS \\
\hline B43 & Ames 13218 & Bolivia, La Paz & USDA-NPGS \\
\hline B44 & Ames 13219 & Bolivia, La Paz & USDA-NPGS \\
\hline C1 & Ames 22153 & Chile, Pichilemu & USDA-NPGS \\
\hline $\mathrm{C} 2$ & Ames 22154 & Chile, Cajon & USDA-NPGS \\
\hline $\mathrm{C} 3$ & Ames 22155 & Chile, Pichaman & USDA-NPGS \\
\hline $\mathrm{C} 4$ & Ames 22156 & Chile, Cajon & USDA-NPGS \\
\hline C5 & Ames 22157 & Chile, Lo Valdivia & USDA-NPGS \\
\hline C6 & Ames 22158 & Chile, Llico & USDA-NPGS \\
\hline $\mathrm{C} 7$ & Ames 22159 & Chile, Bucalemu & USDA-NPGS \\
\hline $\mathrm{C} 8$ & Ames 22160 & Chile, Iloca & USDA-NPGS \\
\hline C9 & Ames 22161 & Chile, Llico & USDA-NPGS \\
\hline C10 & PI 614880 & Chile, Los Lagos & USDA-NPGS \\
\hline C11 & PI 614882 & Chile, La Araucania & USDA-NPGS \\
\hline C12 & PI 614885 & Chile, Bio-Bio & USDA-NPGS \\
\hline C13 & PI 614886 & Chile, Maule & USDA-NPGS \\
\hline C14 & PI 614887 & Chile, Bio-Bio & USDA-NPGS \\
\hline C15 & PI 614888 & Chile, Bio-Bio & USDA-NPGS \\
\hline C16 & PI 614889 & Chile, Bio-Bio & USDA-NPGS \\
\hline $\mathrm{C} 17$ & PI 433232 & Chile, Groben & USDA-NPGS \\
\hline C18 & PI 584524 & Chile, Chillan & USDA-NPGS \\
\hline E1 & Ames 13228 & Equador, Otavalo & USDA-NPGS \\
\hline P1 & PI 510532 & Peru, Puno & USDA-NPGS \\
\hline P2 & PI 510533 & Peru, Puno & USDA-NPGS \\
\hline P3 & PI 510536 & Peru, Puno & USDA-NPGS \\
\hline P4 & PI 510537 & Peru, Puno & USDA-NPGS \\
\hline P5 & PI 510543 & Peru, Puno & USDA-NPGS \\
\hline P6 & PI 510547 & Peru, Puno & USDA-NPGS \\
\hline P8 & PI 510551 & Peru, Puno & USDA-NPGS \\
\hline P9 & PI 596498 & Peru, Cusco & USDA-NPGS \\
\hline P10 & PI 510542 & Peru, Puno & USDA-NPGS \\
\hline P11 & PI 510540 & Peru, Puno & USDA-NPGS \\
\hline P12 & PI 510550 & Peru, Puno & USDA-NPGS \\
\hline P13 & PI 510545 & Peru, Puno & USDA-NPGS \\
\hline P14 & PI 510548 & Peru, Puno & USDA-NPGS \\
\hline P15 & Ames 26191 & Peru, Puno & USDA-NPGS \\
\hline P16 & PI 510546 & Peru, Puno & USDA-NPGS \\
\hline CO1 & PI 596293* & USA, Colorado & USDA-NPGS \\
\hline MD1 & NSL 86628* & USA, Maryland & USDA-NPGS \\
\hline N1 & NSL 91567* & USA, New Mexico & USDA-NPGS \\
\hline N2 & Ames 13719* & USA, New Mexico & USDA-NPGS \\
\hline N3 & Ames $13721^{*}$ & USA, New Mexico & USDA-NPGS \\
\hline N4 & Ames $13722^{*}$ & USA, New Mexico & USDA-NPGS \\
\hline N5 & Ames 13723* & USA, New Mexico & USDA-NPGS \\
\hline N6 & Ames 13724* & USA, New Mexico & USDA-NPGS \\
\hline N7 & Ames 13726* & USA, New Mexico & USDA-NPGS \\
\hline N8 & Ames $13727^{*}$ & USA, New Mexico & USDA-NPGS \\
\hline N9 & Ames 13728* & USA, New Mexico & USDA-NPGS \\
\hline N10 & Ames 13729* & USA, New Mexico & USDA-NPGS \\
\hline N11 & Ames 13730* & USA, New Mexico & USDA-NPGS \\
\hline N12 & Ames 13731* & USA, New Mexico & USDA-NPGS \\
\hline N14 & Ames 13733* & USA, New Mexico & USDA-NPGS \\
\hline N15 & Ames $13734^{*}$ & USA, New Mexico & USDA-NPGS \\
\hline N16 & Ames 13735* & USA, New Mexico & USDA-NPGS \\
\hline N17 & Ames $13736^{*}$ & USA, New Mexico & USDA-NPGS \\
\hline N18 & Ames 13737* & USA, New Mexico & USDA-NPGS \\
\hline
\end{tabular}


Table 1. Continued

\begin{tabular}{|c|c|c|c|}
\hline Sample ID/name & Accession no. & Passport/origin $^{\mathrm{a}}$ & Source $^{b}$ \\
\hline N19 & Ames 13738* & USA, New Mexico & USDA-NPGS \\
\hline N20 & Ames 13739* & USA, New Mexico & USDA-NPGS \\
\hline N21 & Ames 13740* & USA, New Mexico & USDA-NPGS \\
\hline N22 & Ames $13741^{*}$ & USA, New Mexico & USDA-NPGS \\
\hline N23 & Ames $13742^{*}$ & USA, New Mexico & USDA-NPGS \\
\hline $\mathrm{N} 24$ & Ames 13743* & USA, New Mexico & USDA-NPGS \\
\hline N25 & Ames 13744* & USA, New Mexico & USDA-NPGS \\
\hline N26 & Ames $13745^{*}$ & USA, New Mexico & USDA-NPGS \\
\hline $\mathrm{N} 27$ & Ames $13746^{*}$ & USA, New Mexico & USDA-NPGS \\
\hline N28 & Ames $13747^{*}$ & USA, New Mexico & USDA-NPGS \\
\hline N29 & Ames 13748* & USA, New Mexico & USDA-NPGS \\
\hline N30 & Ames 13749* & USA, New Mexico & USDA-NPGS \\
\hline N31 & Ames $13750^{*}$ & USA, New Mexico & USDA-NPGS \\
\hline N32 & Ames $13751^{*}$ & USA, New Mexico & USDA-NPGS \\
\hline N34 & Ames $13753^{*}$ & USA, New Mexico & USDA-NPGS \\
\hline N35 & Ames 13754* & USA, New Mexico & USDA-NPGS \\
\hline N36 & Ames $13755^{*}$ & USA, New Mexico & USDA-NPGS \\
\hline N37 & Ames 13756* & USA, New Mexico & USDA-NPGS \\
\hline N38 & Ames 13757* & USA, New Mexico & USDA-NPGS \\
\hline N39 & Ames 13758* & USA, New Mexico & USDA-NPGS \\
\hline N40 & Ames 13759* & USA, New Mexico & USDA-NPGS \\
\hline N41 & Ames 13760* & USA, New Mexico & USDA-NPGS \\
\hline N42 & Ames $13761^{*}$ & USA, New Mexico & USDA-NPGS \\
\hline N43 & Ames $13762^{*}$ & USA, New Mexico & USDA-NPGS \\
\hline N44 & Ames $13725^{*}$ & USA, New Mexico & USDA-NPGS \\
\hline N45 & Ames $13720^{*}$ & USA, New Mexico & USDA-NPGS \\
\hline SC1 & NSL 86649* & USA, South Carolina & USDA-NPGS \\
\hline TX1 & Ames 19047* & USA, Texas & USDA-NPGS \\
\hline WA1 & NSL 92331* & USA, Washington & USDA-NPGS \\
\hline Cal & PI 433378 & USA, lowa, C. album & USDA-NPGS \\
\hline M1 & PI 476820 & Mexico, C. berlandieri & USDA-NPGS \\
\hline Cgig & Ames 19046 & India, C. giganteum & USDA-NPGS \\
\hline
\end{tabular}

a Source of materials was determined from the USDA Germplasm Resources Information Network (GRIN). Accessions lacking specific passport data are denoted with an asterisk and were assigned the US state from which they were donated as their source ID. Accessions donated to the USDA by Emigdio Ballón (Ballón collection) were assigned the source ID 'USA, New Mexico'.

${ }^{\mathrm{b}}$ USDA-NPGS, USDA North Central Regional Plant Introduction Station of the US National Plant Germplasm System (Ames, IA); CIP-FAO, Centro Internacional de la Papa; PROINPA, The Foundation for the Promotion and Investigation of Andean Products.

\section{Results}

\section{Microsatellite diversity}

A total of 420 alleles were identified by the 36 microsatellite loci among the 152 quinoa accessions analysed (control species were not included in the analysis). The number of alleles per locus ranged from three (QAAT10) to 27 (QAAT50 and QAAT76) with an average of 11.7 alleles per locus (Table 2). The tri-nucleotide repeat primers amplified a significantly greater number of alleles ( $t$-test; $P \leq 0.05$ ) per locus (average $15.2 ; n=15$ ) than did the di-nucleotide repeats (average $9.1 ; n=21)$.
Heterozygosity $(H)$ values for the microsatellite loci ranged from 0.45 to 0.94 with a mean value of 0.75 . As expected, the mean $H$ value in this experiment is larger than the average $H$ value (0.57) reported by Mason et al. (2005). The increase in the average heterozygosity value in this study is likely due to: (i) the inclusion of only highly informative markers; (ii) better resolution of marker alleles (single base pair resolution) due to the use of capillary electrophoresis; and (iii) the screening of a much larger and likely more diverse panel of genotypes. All of these factors should lead to an increase in number of alleles observed and consequently increased $H$ values. According to the definition of Ott (1992), markers are considered polymorphic if $H \geq 0.1$ and highly polymorphic if $H \geq 0.7$, thus all of the 
Table 2. Number of alleles, number of rare alleles, heterozygosity value, primer sequences and GenBank accession number for each microsatellite marker used to $₫$ assess genetic variation in 150 accessions of quinoa

\begin{tabular}{|c|c|c|c|c|c|c|c|c|}
\hline Primer & $\begin{array}{c}\text { Fluorescent } \\
\text { label }\end{array}$ & $\begin{array}{l}\text { No. of } \\
\text { alleles }\end{array}$ & $\begin{array}{l}\text { No. of } \\
\text { rare alleles }\end{array}$ & $H$ value & $\begin{array}{c}\text { Allelic } \\
\text { size range (bp) }\end{array}$ & Forward primer $\left(5^{\prime}-3^{\prime}\right)$ & Reverse primer $\left(5^{\prime}-3^{\prime}\right)$ & $\begin{array}{c}\text { GenBank } \\
\text { accession no }\end{array}$ \\
\hline KGA03* & HEX & 21 & 15 & 0.85 & $140-182$ & HEX-attgccgacaatgaacgaat & gcttctatgtaaatggcatgtccсаac & DQ462129 \\
\hline KGA16* & 6FAM & 9 & 3 & 0.76 & $155-196$ & 6FAM-ccctgcttaatctccgtgaa & gcttctccgaaccaagactacgaaaca & DQ462130 \\
\hline KGA20* & NED & 17 & 7 & 0.87 & $155-185$ & gcttcttcacctacctcggtaaaggaaa & NED-ggagcagatgatgaacatgg & DQ462131 \\
\hline KGA27* & NED & 16 & 8 & 0.90 & $126-158$ & NED-ttgtacagaggaagtggcaaga & gcttctcatcttacagctctggetttcc & DQ462132 \\
\hline QAAT06* & 6FAM & 12 & 5 & 0.81 & $193-226$ & gcttctcacaaacaataattcaaccgaaga & 6FAM-cgctgacgcttaacattcg & DQ462133 \\
\hline QAAT10 & 6FAM & 3 & 0 & 0.45 & $148-161$ & gcttcttgaaatcgaaacggaggaag & 6FAM-gggataggactgatgccaga & DQ462134 \\
\hline QAAT22* & $6 \mathrm{FAM}$ & 26 & 15 & 0.94 & $153-235$ & gcttcttggtcgatatagatgaaccaaa & 6FAM-ggagcccagattgtatctca & DQ462135 \\
\hline QAAT24 & 6FAM & 20 & 13 & 0.85 & $201-257$ & gcttctaccataacagcacccacctt & 6FAM-agggatcaatcttgttcattca & DQ462136 \\
\hline QAAT50* & $\mathrm{VIC}$ & 27 & 19 & 0.89 & $158-246$ & VIC-ggcacgtgctgctactcata & gcttctatggcgaatggttaatttgc & DQ462137 \\
\hline QAAT69* & NED & 15 & 7 & 0.88 & $193-266$ & NED-gtttcctttgaggcttggac & gcttctggatttgtacgaatagttgggatt & DQ462138 \\
\hline QAAT70* & NED & 17 & 6 & 0.91 & $158-208$ & NED-tgaacaggatcgtcatagtcaa & gcttctcgttcatcatctgacccaat & DQ462139 \\
\hline QAAT71* & TET & 25 & 14 & 0.92 & $122-200$ & TET-catcacccgctgaatagacac & gcttcttaccctaatgccacgattcc & DQ462140 \\
\hline QAAT74* & 6FAM & 16 & 7 & 0.87 & $169-224$ & gcttctatggaacacccatccgataa & 6FAM-atgcctatcctcatcctcca & DQ462141 \\
\hline QAAT76* & HEX & 27 & 17 & 0.92 & $145-227$ & HEX-gcttcatgtgttataaaatgccaat & gcttcttctcggcttcccactaatttt & DQ462142 \\
\hline QAAT78* & HEX & 13 & 4 & 0.85 & $183-226$ & HEX-agcgaaggaaatttggaact & gcttcttaacgatacgctccaaggaa & DQ462143 \\
\hline QATG19 & 6FAM & 7 & 2 & 0.74 & $175-193$ & gcttctccaaacaaagacaataaggaaacc & 6FAM-cgaggttgaaggagattcca & DQ462144 \\
\hline QATG52 & 6FAM & 11 & 4 & 0.73 & $200-253$ & 6FAM-tgcagtgaagtgaacaagagaga & gcttctgcaccaaatcacaccattca & DQ462145 \\
\hline QATG60 & 6FAM & 4 & 1 & 0.59 & $174-189$ & gcttcttggtgaaagcagttatgaagaca & 6FAM-tttcagctcaacctagaacacg & DQ462146 \\
\hline QATG86 & VIC & 5 & 1 & 0.61 & $189-200$ & VIC-aatcgcagcctaaactgagc & gcttctagttccatttcgaccatatgataa & DQ462147 \\
\hline QCA06 & $6 \mathrm{FAM}$ & 6 & 3 & 0.56 & $109-127$ & 6FAM-gctctattaaggaaatgaggttaca & gcttctgccattcaattcagcaaagg & DQ462148 \\
\hline QCA11* & VIC & 4 & 0 & 0.60 & $199-206$ & VIC-caggagcctcaagaatgaca & gcttcttttaaattcaagcctaccaccttt & DQ462149 \\
\hline QCA14 & VIC & 8 & 2 & 0.59 & $196-211$ & gcttctcctgagctgatttatcaaaggac & VIC-cctcttgcgagatttctgct & DQ462150 \\
\hline QCA19* & VIC & 6 & 2 & 0.69 & $183-212$ & VIC-tttcatcactcgaccgtatagc & gcttctagggtgactgttacacccaaa & AY458236 \\
\hline QCA24* & VIC & 7 & 2 & 0.75 & $235-254$ & VIC-agatgagcttgaatcattacatc & gcttcttacatactgtaaatcatgccaaa & DQ462151 \\
\hline QCA37* & $\mathrm{VIC}$ & 9 & 1 & 0.77 & $186-206$ & gcttctccgttcttccagaccaattc & VIC-tcatgagccacttcatacacg & AY458227 \\
\hline QCA38* & VIC & 5 & 1 & 0.71 & $198-209$ & gcttctcatttcccaaactgcatgaat & VIC-atgtgtgttgcgtgtgagtg & DQ462152 \\
\hline QCA48* & 6FAM & 6 & 2 & 0.65 & $246-258$ & gcttctacaatacatacataacccaatattcaa & 6FAM-tggaaatgtcactatgattgga & AY458240 \\
\hline QCA57* & NED & 8 & 2 & 0.78 & $168-189$ & gcttcttgcaaggaaaccatctttgg & NED-tgcctcacagtcacacctaca & AY458243 \\
\hline QCA58* & NED & 8 & 2 & 0.80 & $170-193$ & NED-ctcgaccagcagggtctg & gcttctctagctaggcgttgcctgac & AY458244 \\
\hline QCA65* & NED & 9 & 3 & 0.79 & $168-206$ & gcttctccatgcaagggaacatattg & NED-aagttcgttggcttgctgta & AY458246 \\
\hline QCA71* & $6 \mathrm{FAM}$ & 16 & 10 & 0.84 & $140-177$ & 6FAM-aacaacgaaattacgagaatgtca & gettcttctcacgagagtcttccceta & DQ462153 \\
\hline QCA88 & NED & 8 & 6 & 0.49 & $126-246$ & gcttcttctggctgcttccacctaat & NED-cagtcccggaatcgtaactc & DQ462154 \\
\hline QCA107* & NED & 5 & 1 & 0.70 & $153-167$ & gcttctacaggctgtgggtccactt & NED-tcaagcaatactcaccttgtgg & DQ462155 \\
\hline Q̀CA120 & NED & 6 & 0 & 0.69 & $171-198$ & gcttctgacgcacataacgtgtagaattg & NED-tcctcattccettctccatc & DQ462156 \\
\hline QGA03* & NED & 10 & 3 & 0.79 & $188-209$ & gcttctgaacctttaataggtctgtaccaaatc & NED-aagaaatgtcacaagcaagca & DQ462157 \\
\hline QGA17 & NED & 8 & 5 & 0.52 & $145-164$ & NED-ttacggttctcccggtctc & gcttcttgcaaacaagagaagcatgaag & DQ462158 \\
\hline Mean & & 12 & 5 & 0.75 & & & & \\
\hline
\end{tabular}

A total of 420 alleles were amplified across the $C$. quinoa genotypes ranging from three to 27 alleles per locus (mean $=11.7$ ). Primers that differed for the most common allele between the lowland and highland groups are denoted with an asterisk. Bolded portions of the primer sequences represent the nucleotides (gcttct) added to the primer to facilitate PCR. $\infty$ 
microsatellite markers utilized in this study were polymorphic and 25 (69\%) were highly polymorphic.

The allelic size range variation (defined as base pairs from smallest to largest allele) at a microsatellite locus was highly correlated $(P \leq 0.01)$ with the total number of alleles identified per locus ( $r=0.71$; Table 2$)$. Since different microsatellite loci carrying the same fluorescent label can be simultaneously analysed in the same gel lane/capillary column if their allele size ranges do not overlap, the determination of the full allelic size range for these microsatellite markers represents an important step towards the future development of multiplex marker sets based on allele size ranges. Such multiplexed sets will dramatically reduce the cost associated with genotyping. Several researchers have reported the development of six- and nine-plex marker systems for a wide variety of crop species including soybean (Diwan and Cregan, 1997), sunflower (Tang et al., 2003) and rape seed (Tommasini et al., 2003). In the present study, the average allelic range across the microsatellites was 37 bp (Table 2).

\section{Rare and high-frequency microsatellite alleles}

Rare alleles, defined as those that were observed in less than $5 \%$ of the accessions were detected at all loci, except QAAT10, QCA11 and QCA120 (Table 2). QAAT50 displayed the highest number of alleles (27), and the highest percentage $(70 \%)$ of rare alleles (19/27 alleles; Table 2$)$. Not unexpectedly, and as seen by other researchers (Jain et al., 2004), a positive significant correlation existed between the number of rare alleles and the number of alleles per locus $(r=0.96)$. Rare alleles are of particular value for variety identification via DNA fingerprinting.

High-frequency alleles (common alleles) were seen within the majority of the loci analysed. Indeed, on average 76 (50\%) of the 152 accessions shared a common allele at any given locus. A negative correlation between the frequency of the most common allele at a locus and the number of alleles per locus was observed $(r=-0.78)$. Within the group of 81 highland accessions the average frequency of shared alleles was 53\%, whereas within the group of 71 lowland (including the Ballón collection) ecotypes the frequency of shared alleles rose to $61 \%$, suggesting a lower level of genetic diversity within the coastal ecotypes. Moreover, a comparison of the most common allele observed in the highland and lowland ecotype groups indicated that the most common allele differed between the groups in 25 of the 36 microsatellite loci (microsatellite markers that differed for the most common alleles between the two groups are marked with an asterisk in Table 2). Based on the highest allele frequency alone, the two groups could be distinguished from each other at $69 \%$ of the microsatellite loci.

\section{Heterogeneity}

Genetic heterogeneity, detected as multiple alleles at a genetic locus within an individual accession (allele mixtures), was observed on average in $32 \%$ of the quinoa accessions per microsatellite locus. The presence of heterogeneity in the quinoa accessions was not unexpected and may be explained by low levels of residual heterozygosity at individual loci due to outcrossing during seed maintenance (outcrossing estimates range from 8 to 10\%; A. Bonifacio, personal communication) or as a result of seed mixtures of contrasting homozygous (or heterozygous) lines inherent to the heterogeneous quinoa seed lots, which would be detected as a result of the leaf-bulking strategy utilized in the DNA preparation of each accession. Indeed, phenotypic inspection of field-grown seed lots revealed low levels of phenotypic diversity in many of the accessions utilized in these experiments, suggesting the presence of genetic heterogeneity within accessions and the need for future characterization of within-line genetic diversity. Loci that produced multiple alleles were scored as having all alleles present.

\section{Phenetic analysis and genetic similarities}

Jaccard's similarity coefficients for quinoa accessions ranged from 0.038 to 1 , with the least genetic similarity detected between the Bolivian highland accession B15 (PI614002) and the lowland Chilean accession C15 (PI614888), whereas the greatest genetic similarity was observed between the genetically identical lowland accessions WA (NSL92331) and SC (NSL86649). Distinct genetic profiles were identified for all other accessions.

The UPGMA and Jaccard's similiarity coefficients were used to produce a dendrogram of quinoa accessions and the two control Chenopodium control species (Fig. 1). C. album and C. giganteum clustered at the root of the UPGMA dendrogram with the Mexican accession M1 (Fig. 1; see Discussion). This Mexican accession (M1) was extremely distinct from all other quinoa accessions as shown by it low pairwise similarity value (0.073) and was thus removed from subsequent analyses. The remaining 150 quinoa accessions, clustered into two large, but clearly distinct, clusters corresponding to the highland Andean and lowland coastal accessions (Fig. 1). The highland cluster consisted of accessions from Peru, Bolivia, Ecuador and Argentina and are broken down into two subgroups: northern highland and southern highland. All four of the Ecuadorian accessions clustered together with all but four (P11, P15, P6 and $\mathrm{P} 17$ ) of the Peruvian accessions in the northern highland subgroup, whereas all but three Bolivian accessions 
(B37, B38 and B39) clustered with the majority of the Argentinian accessions in the southern highland subgroup (Fig. 1). One Argentinian accession (A2) clustered with the coastal lowland accessions. The Chilean accessions all clustered in the coastal lowland group with the exception of 'Ollague', a highland ecotype originating from northern Chilean Altiplano. Most of the USDA-Ballón collection clustered in the lowland coastal

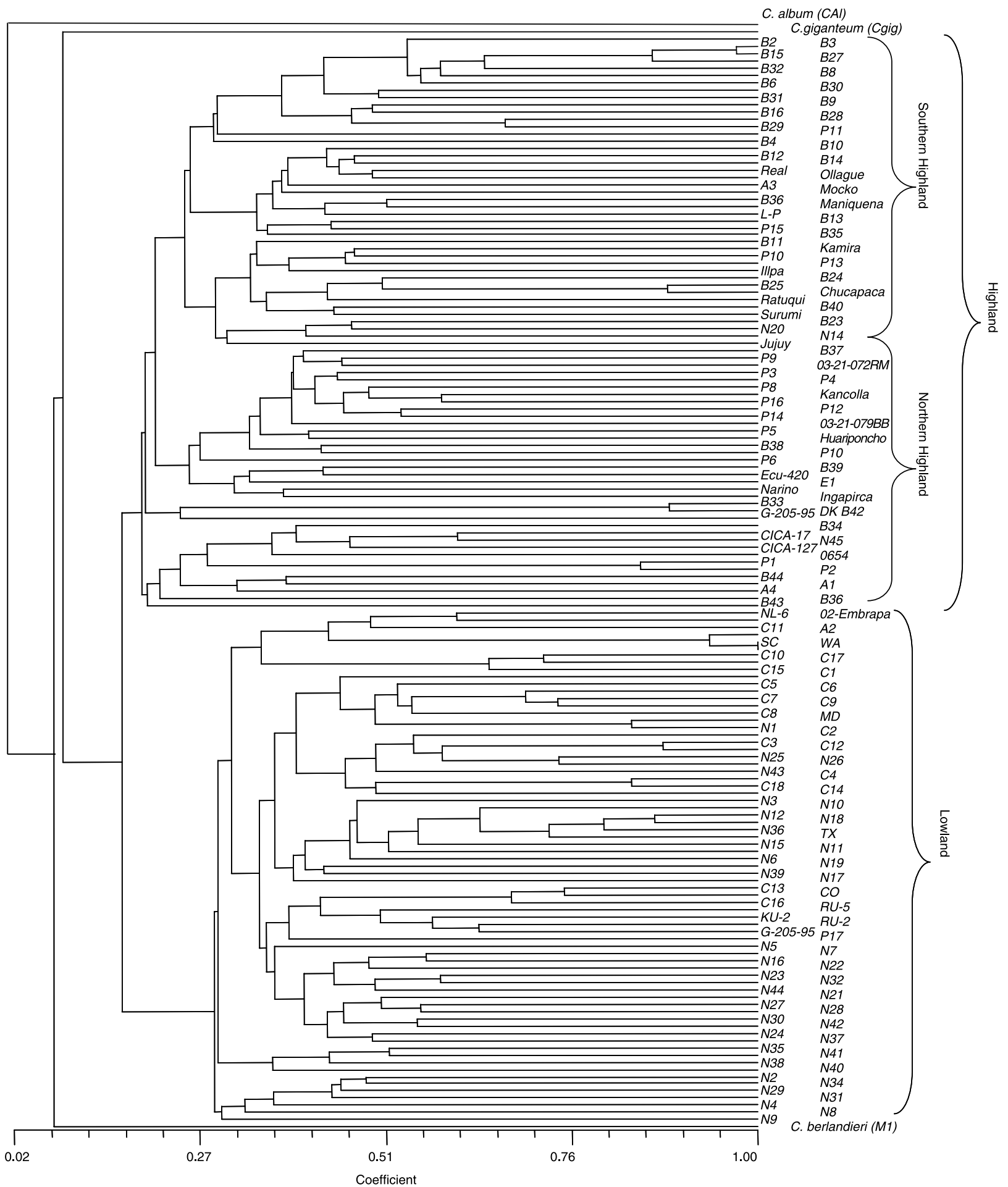

Fig. 1. Dendrogram showing the 150 accessions of Chenopodium quinoa, one accession of C. berlandieri Moq. ssp. nuttaliae (M1) and the two control species (C. album-Cal; C. giganteum-Cgig), from UPGMA cluster analysis using allelic data from 36 microsatellite markers. C. quinoa accessions are clustered into two main groups: highland (top) and lowland (bottom) ecotypes. 
group, with the exception of N20 and N14 which grouped with the accessions in the southern highland subgroup (Table 1; Fig. 1).

PCA formed two well-separated groups (highland and lowland; Fig. 2). The plot of PCA Dim-1 against Dim-2 accounted for $21.8 \%$ (Dim-1 $=15.9 \%$; Dim- $2=5.9 \%$ ) of the total variation observed in the 152 quinoa accessions analysed. The lowland cluster forms a single continuum along the Dim-1 axis. Within this continuum, the Chilean lowland and Ballón accessions segregate to the opposite extremes, with the Ballón accessions stretching towards the highland cluster (Fig. 2). No discrete breaks occur between the Chilean and Ballón accessions. The highland variation forms a single continuum, easily seen along the Dim-2 axis, with northern (Ecuadorian and Peruvian) and southern (Bolivian and Argentinian) accessions stretching to opposite extremes of the Dim-2 axis. Some Peruvian (northern highland) and Bolivian (southern highland) accessions intermingle within the centre of the highland cluster, preventing the separation of northern and southern highland accessions into discrete entities.

Pairwise comparisons of the similarity coefficient within the highland group (mean $=0.23$ ) are significantly lower ( $t$-test; $P \leq 0.01$ ) than comparisons within the lowland group (mean $=0.34$ ), suggesting that the lowland group contains less genetic diversity. Table 3 presents the average similarity value between accessions based on country of origin (or collector in the case of the Ballón collection). The highest similarities between countries were observed between the Chilean genotypes and the Ballón collection (0.31). In contrast, the greatest dissimilarity between countries was observed between the Chilean and Ecuadorian genotypes, followed closely by the comparison of the Chilean and Peruvian genotypes (0.12 and 0.13, respectively). Interestingly, the genetic similarity comparisons between the Ballón collection and Peruvian and

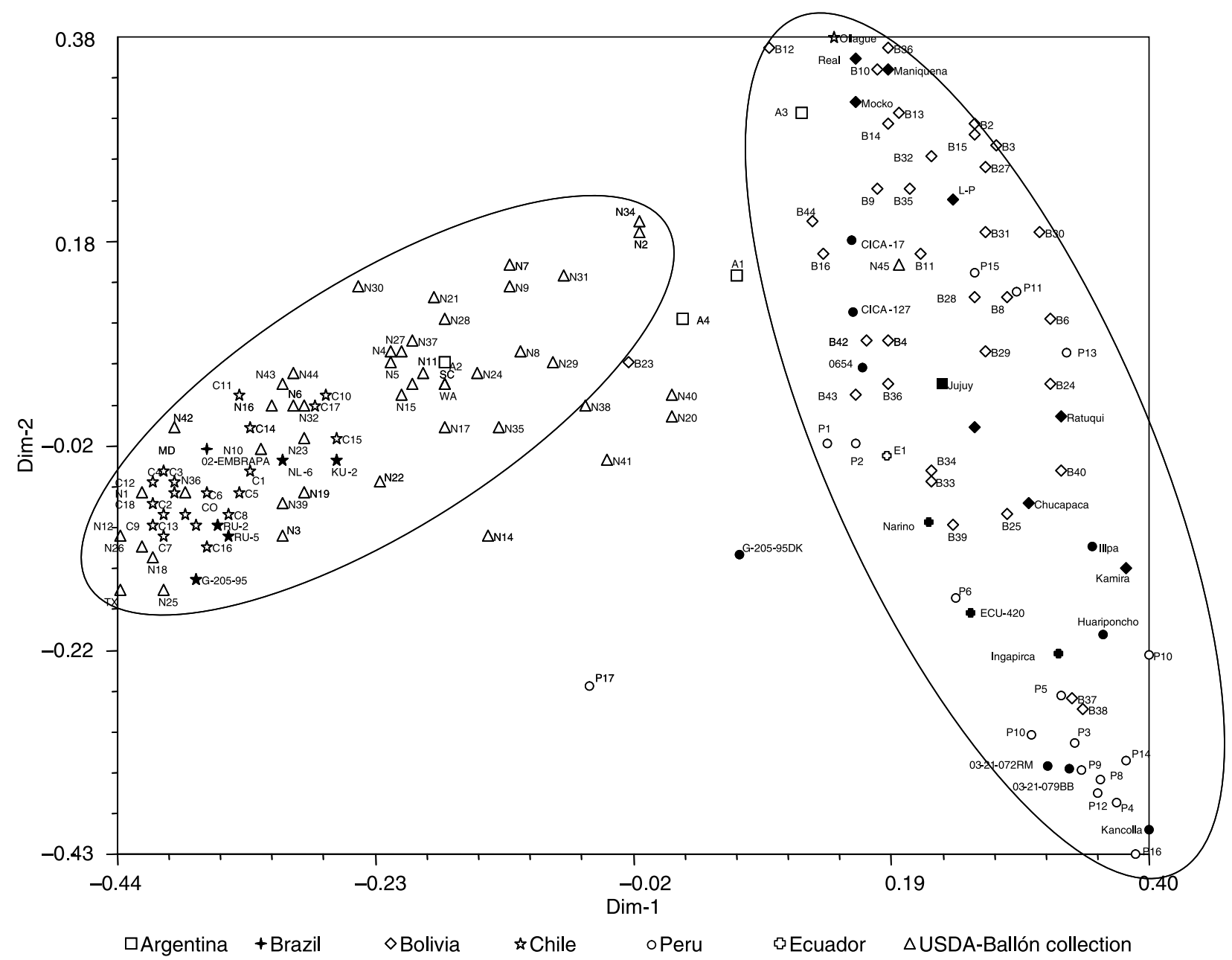

Fig. 2. The two-dimensional scaling resulting from the PCA analysis of 150 Chenopodium quinoa accessions using genetic diversity data from 36 microsatellite markers. Circled areas represent the major highland and lowland clusters identified in the dendrogram. Accessions are coded for their country of origin as described in the legend. Blackened symbols indicate accessions from CIP-FAO and PROINPA collections. 
Table 3. Total alleles, mean number of alleles per region, unique alleles and pairwise similarity comparisons given for each country represented in the analysis (the single quinoa accession from Brazil (02-Embrapa) is not included in the analysis)

\begin{tabular}{|c|c|c|c|c|c|c|}
\hline & $\begin{array}{c}\text { Ecuador } \\
(n=4)\end{array}$ & $\begin{array}{c}\text { Peru } \\
(n=28)\end{array}$ & $\begin{array}{c}\text { Bolivia } \\
(n=42)\end{array}$ & $\begin{array}{c}\text { Argentina } \\
(n=5)\end{array}$ & $\begin{array}{c}\text { Chile } \\
(n=24)\end{array}$ & $\begin{array}{l}\text { Ballón } \\
\text { collection } \\
(n=48)\end{array}$ \\
\hline \multicolumn{7}{|l|}{ Allelic diversity } \\
\hline Total alleles & 131 & 271 & 290 & 162 & 198 & 241 \\
\hline Mean no. of alleles per locus & 3.6 & 7.5 & 8.1 & 4.5 & 5.5 & 6.7 \\
\hline Unique alleles & 5 & 13 & 17 & 10 & 9 & 4 \\
\hline \multicolumn{7}{|c|}{ Pairwise similarity comparisons among countries } \\
\hline Ecuador & 0.337 & & & & & \\
\hline Peru & 0.243 & 0.274 & & & & \\
\hline Bolivia & 0.200 & 0.212 & 0.262 & & & \\
\hline Argentina & 0.165 & 0.171 & 0.209 & 0.208 & & \\
\hline Chile & 0.125 & 0.133 & 0.144 & 0.199 & 0.350 & \\
\hline Ballón collection & 0.159 & 0.167 & 0.185 & 0.209 & 0.309 & 0.333 \\
\hline
\end{tabular}

a The Ballón collection lack specific passport information regarding country of origin. According to the phenetic analysis they are more similar to the lowland accessions originating from Chile.

Bolivian genotypes were intermediate $(0.19$ and 0.17 , respectively) as compared to the distances between the Chilean material and the Peruvian and Bolivian genotypes (0.13 and 0.14 , respectively).

\section{Discussion}

Phenetic analyses of quinoa accessions show that the lowland and highland accessions segregate into two discrete clusters consistent with Wilson's (1988a) hypothesis. Additionally, the highland cluster segregates into northern (northern Peru/Ecuador) and southern (southern Peru/Bolivia/northern Argentina) highland subgroups (Fig. 2). Gandarillas (1979) and Wilson (1988b) suggested that the southern highlands region near Lake Titicaca represents the centre of diversity for quinoa based on the high levels of morphological and isozyme diversity observed in this region. Diversity measures based on the molecular data reported herein support this view since the regions to the north and south of this region show a decrease in genetic variation (Table 3). The reduction in genetic diversity levels in the northern highland and coastal lowland regions could be a result of founder effects associated with the dispersal of the crop from its centre of origin or alternatively as a response to selective adaptation in the more uniform ecological regions of the northern highlands and coastal lowlands. Given quinoa's ancient association with the Incan civilization any interpretation of quinoa's spread through South America needs to consider the broad implications of human intervention (Wilson, 1988a).

Two hypotheses (Gandarillas, 1979; Tapia, 1979; Wilson, 1988a) have been proposed to explain the origin of quinoa domestication: (i) quinoa originated in
Colombia and spread southwards along the Andes; and (ii) quinoa originated in the southern highlands of Peru and Bolivia. As Gandarillas (1979) noted, the evidence in support of a northern origin are tenuous at best, as it appears to be mostly anecdotal and linguistic in inspiration (Tapia, 1979). Gandarillas (1979) noted a Peruvian-Bolivian centre of morphological diversity and subsequently proposed the Peruvian and Bolivian southern highlands as the centre of domestication. Our data support the Peruvian-Bolivian centre of diversity; however the lack of accessions from Ecuador and Colombia in the USDA collection precludes the elimination of a northern hypothesis of the origin of domestication of quinoa and further suggests that concentrated collections of quinoa accessions are needed from these countries.

The phenetic analysis showed an interdigitation of Peruvian and Bolivian accessions in both the UPGMA dendrogram and in the PCA analysis, which is likely due to the geographic proximity of some genotypes along the border of Bolivia and Peru on the southern Andean Altiplano. Ecuadorian accessions form a single group within the northern highland subgroup of the cluster analysis, although they are somewhat scattered within the northern subgroup (Fig. 2), suggesting that the Ecuadorian accessions were likely introduced from the Andean highland group followed by subsequent adaptation. Interestingly, the Argentinian accessions are scattered with no discernible pattern within the dendrogram (Fig. 1) or the PCA (Fig. 2), with one accession (A4) even grouping with coastal Chilean lowland accessions. Although our understanding of Argentinian germplasm is limited by the small sample size available to us in the USDA collection, it does appear that the Argentinian accessions included in this study potentially represent reintroductions of quinoa into Argentina from 
both the southern highland and lowland genotypes. Future systematic and phyletic analysis of quinoa would undoubtedly benefit from the inclusion of a wider array of Argentinian accessions collected from indigenous populations.

Tapia et al. (1980) classified highland quinoa into four distinct ecotypes, Valle, Yungas, Altiplano and Salares, based on altitude, height of the plants, amount of branching, length of growing period and salt tolerance. Wilson (1988a) noted that these classifications were not well supported by isozyme data. Other than the tendency of the Salares types to cluster together along Dim-2, we could detect no correlation between these ecotypes and genetic diversity as revealed in our phenetic analyses. Furthermore, we observed no significant correlation $(P \leq 0.05)$ of genetic diversity using PCA Dim-1 or Dim-2 with altitude, latitude or longitude (data not shown), although we note these correlations were hindered by a lack of reliable passport data for many of the accessions (Pratt and Clark, 2001).

Examination of the lowland cluster shows evidence of two subgroups, the first from lowland Chile and the second made up of populations collected by the Bolivian agronomist Emigdio Ballón. The Chilean subgroup is less diverse as compared to the Peruvian and Bolivian populations (Table 3). The lack of genetic diversity in the Chilean populations supports Wilson's (1988a, b) hypothesis of an ancient founder effect followed by long periods of drift in Chilean populations. $t$-Tests of Jaccard's similarity measures between Chile and Bolivia as well as Chile and Peru showed that the reduction of variation in Chile is statistically significant $(P \leq 0.001)$ for both comparisons. Wilson (1988a) additionally hypothesized that Chilean populations originated from the southern Andes. This hypothesis is further supported by pairwise comparisons of the Jaccard's similarity values based on country of origin which indicated that Chilean populations are more similar to the southern highland genotypes from Argentina (0.199) and Bolivia (0.144) than they are to the northern highland genotypes from Peru (0.133) and Ecuador (0.125).

An anomaly in the data is the Mexican accession M1 (PI 476820, 'Santa Elena 7') which grouped by itself in the phenetic analysis and was genetically distinct from all other quinoa accessions, with a substantially reduced average genetic similarity value of 0.073 (7.3\%) when compared to all other quinoa accessions. The USDA's Germplasm Resources Information Network (GRIN) lists M1 as C. quinoa, but also lists 'huazontle' as an additional name for this accession. Huazontle is the common name used to refer to $C$. berlandieri Moq. ssp. nuttalliae, a cultivated chenopod grown in central Mexico. Nelson (1968) refers specifically to 'Santa Elena \#7' as C. nuttalliae Safford, a former classification for
C. berlandieri ssp. nuttalliae. Huazontle and quinoa are both members of the series Foveosa (subsect Cellulata, sect Chenopodium), and the two cross readily. Aellen (1929) treated the two as conspecific. They are separated geographically and distinguished by slight differences in leaf shape and seed pigmentation (Simmonds, 1965; Nelson, 1968). M1 is from within the range of huazontle, and well outside the area where C. quinoa is commonly found. Hence, the data presented here suggest that M1 is misclassified as C. quinoa and should be reclassified as C. berlandieri Moq. ssp. nuttaliae.

\section{USDA-Ballón collection subset}

Many of the accessions available in the USDA collection were collected by Emigdio Ballón. Unfortunately, the GRIN database lacks passport information for the vast majority of these genotypes. The UPGMA dendrogram clustered the majority ( $>90 \%$ ) of the Ballón genotypes with the Chilean lowland accessions (Fig. 1). The PCA analysis (Fig. 2) also places them within the Chilean lowland group, however the Ballón collection is oriented between the Chilean populations and the southern highland populations, indicating a potential affinity with southern highland accessions that is further supported by pairwise comparisons of Jaccard's similarity values (Table 3).

Shared allelic patterns between the Ballón, Chilean lowland and highland accessions are particularly interesting. Highland, Chilean and the Ballón accessions share a common predominant allele at $31 \%$ of the loci. However, at $44 \%$ of the loci, the Ballón and Chilean accessions shared a predominant allele while the highland accessions exhibited a different predominant allele. In contrast, the Ballón and highland accessions shared a predominant allele at only $7 \%$ of the marker loci. At the remaining loci (18\%) the Ballón accessions have a predominant allele that differs from the predominant allele identified in the highland and Chilean lowland groups. The Ballón collections also show higher diversity measures and they harbour a number of alleles not found in the lowland accessions but present in highland accessions. Thus the Ballón collection appears to be coastal Chilean in terms of predominant alleles, but further enriched by highland alleles.

One intriguing hypothesis that may explain the intermediate position of the Ballón collection is that it may represent a distinct geographic ecotype. One potential geographic area to which this collection could belong is the Yungas region (Tapia et al., 1980), which stretches along the eastern slopes of the Bolivian Andes. Evidence in support of this hypothesis includes: (i) the intermediate position of the Ballón populations along Dim-1 of the 
PCA; (ii) diversity values that are intermediate between Chilean and highland values; (iii) intermediacy of the similarity values for the Ballón accessions as compared to the other geographical regions; and (iv) patterns of predominant alleles. We note that Wilson (1988a) included a single representative of the Yungas ecotype in his isozyme analysis which he reported was distinctive. However, this ecotype is not present in the USDA collection and was, therefore, unavailable for inclusion in this study. If the Ballón collection represents a distinct geographical ecotype, the lack of similarly clustering accessions from the CIP-FAO international nursery collection suggests that it is potentially underrepresented in even the international quinoa germplasm collections. Tracing the origins of the Ballón collection should be a primary objective for the curation efforts of the USDA quinoa germplasm.

\section{Conclusions}

The results of this study hold several implications for quinoa conservation and improvement programmes. Multiple alleles were observed at marker loci for several of the accessions utilized in the study, suggesting that quinoa accessions (both those held by the USDA and those in the CIP-FAO collection) represent potentially heterogeneous lines of mixed genotypes that should be carefully screened and potentially purified prior to breeding. The lack of passport and morphological data associated with many of the publicly available quinoa accessions in the USDA germplasm system may potentially hinder their utility in traditional breeding programmes. We note, however, that extensive collections that are well documented are available in the Peruvian, Bolivian and Chilean germplasm collections. Application of these microsatellite markers to these South American germplasm collections will further elucidate the full range of genetic diversity within C. quinoa. Additionally, this study highlights the need for increased collection from two specific geographical areas: the northern Andes (especially Ecuador and Colombia) and the Yungas (eastern slopes of the Bolivian and Peruvian Andes).

\section{Acknowledgements}

This research was supported by grants from the McKnight Foundation, Holmes Family Foundation, and the Erza Taft Benson Agriculture and Food Institute. We gratefully acknowledge D. Brenner at USDA-NPGS, Iowa State University, for taxonomic suggestions and seed contribution. We thank R. Holmes and M. Dilg for technical assistance with the microsatellite assays.

\section{References}

Aellen P (1929) Beitrag zur systematic der Chenopodium-Arten Amerikas, vorweigend auf Grund der Sammlung des United States National Museum in Washington, D.C. Feddes Repertorium Specierum Novarum Regni Vegetabilis 26: 31-67, 119-160.

Cusack DF (1984) Quinua: grain of the Incas. Ecologist 14: $21-31$.

Dean RE, Dahlberg JA, Hopkins MS, Mitchell SE and Kresovich S (1999) Genetic redundancy and diversity among 'orange' accessions in the US National Sorghum Collection as assessed with simple sequence repeat (SSR) markers. Crop Science 39: 1215-1221.

Diwan N and Cregan PB (1997) Automated sizing of fluorescentlabeled simple sequence repeat (SSR) markers to assay genetic variation in soybean. Theoretical and Applied Genetics 95: 723-733.

Elder JK and Southern EM (1987) Computer-aided analysis of one dimensional restriction fragment gels. In: Bishop MJ and Rawlings CJ (eds) Nucleic Acid and Protein Sequence Analysis - A Practical Approach. Oxford: IRL Press, pp. 165-172.

Gandarillas H (1979) Genética y orígen. In: Tapia ME (ed.) Quinua y Kañiwa: Cultivos Andinos. Bogota: Instituto Interamericano de Ciencias Agrícolas, pp. 45-64.

Gupta PK and Varshney RK (2000) The development and use of microsatellite markers for genetic analysis and plant breeding with special emphasis on bread wheat. Euphytica 113: $163-185$.

Hokanson SC, Szewc-McFadden AK, Lamboy WF and McFerson JR (1998) Microsatellite (SSR) markers reveal genetic identities, genetic diversity and relationships in a Malus $\times$ domestica Borkh. core subset collection. Theoretical and Applied Genetics 97: 671-683.

Jacobsen SE (2000) Quinoa-research and development at the International Potato Center (CIP). Paper presented at the Annual Meeting of the Consultative Directorate of CONDESAN, November, pp. 1-5 Lima, peru.

Jain S, Jain RK and McCouch Lima, Peru SR (2004) Genetic analysis of Indian aromatic and quality rice (Oryza sativa L.) germplasm using panels of fluorescently-labeled microsatellite markers. Theoretical and Applied Genetics 109: 965-977.

Mace ES and Godwin ID (2002) Development and characterization of polymorphic microsatellite markers in taro (Colocasia esculenta). Genome 45: 823-832.

Mason SL, Stevens MR, Jellen EN, Bonifacio A, Fairbanks DJ, Coleman CE, McCarty RR, Rasmussen AG and Maughan PJ (2005) Development and use of microsatellite markers for germplasm characterization in quinoa (Chenopodium quinoa Willd.). Crop Science 45: 1618-1630.

Maughan PJ, Saghai Maroof MA, Buss GR and Huestis GM (1996) Amplified fragment length polymorphism (AFLP) in soybean: species diversity, inheritance, and near-isogenic line analysis. Theoretical and Applied Genetics 93: 392-401.

McGregor CE, van Treuren R, Hoekstra R and van Hintum TJL (2002) Analysis of the wild potato germplasm of the series Acaulia with AFLPs: implications for ex situ conservation. Theoretical and Applied Genetics 104: 146-156.

Mujica A, Jacobsen SE, Izquierdo J and Marathee JP (1998) Prueba Americana y Europea de quinoa (Chenopodium quinoa Willd.). Puno: FAO, Libro de Campo.

Nelson, DC (1968) Taxonomy and origins of Chenopodium quinoa and Chenopodium nuttalliae. PhD Thesis, Indiana University. 
Ortiz R, Ruiz-Tapia EN and Mujica-Sanchez A (1998) Sampling strategy for a core collection of Peruvian quinoa germplasm. Theoretical and Applied Genetics 96: $475-483$.

Ott J (1992) Strategies for characterizing highly polymorphic markers in human gene mapping. American Journal of Human Genetics 51: 283-290.

Pratt DB and Clark LG (2001) Amaranthus rudis and A. tuberculatus - one species or two?" Journal of the Torrey Botanical Society 128: 282-296.

Risi JC and Galwey NW (1984) The Chenopodium grains of the Andes: Inca crops for modern agriculture. Advances in Applied Biology 10: 145-216.

Roa NK (2004) Plant genetic resources: advancing conservation and use through biotechnology. African Journal of Biotechnology 3: 136-145.

Rohlf FJ (2000) NTSYSPc 2.1: Numerical Taxonomy and Multivariate Analysis System. New York: Exeter Software.

Rojas W, Barriga P and Figueroa H (2000) Multivariate analysis of the genetic diversity of Bolivian quinoa germplasm. Plant Genetic Resources Newsletter 122: 16-23.

Ruales J and Nair BM (1992) Nutritional quality of the protein in quinoa (Chenopodium quinoa, Willd) seeds. Plant Foods and Human Nutrition 42: 1-11.

Ruales J, de Grijalva Y, Lopez-Jaramillo P and Nair BM (2002) The nutritional quality of an infant food from quinoa and its effect on the plasma level of insulin-like growth factor-1 (IGF-1) in undernourished children. International Journal of Food Science and Nutrition 53: $143-154$.

Sambrook J, Fritsch EF and Maniatis T (1989) Molecular Cloning: A Laboratory Manual. 2nd edn. New York: Cold Spring Harbor Press.
Simmonds NW (1965) The grain chenopods of the tropical American highlands. Economic Botany 19: 223-235.

Simmonds NW (1971) The breeding system of Chenopodium quinoa. I. Male sterility. Heredity 27: 73-82.

Tang S, Kishore VK and Knapp SJ (2003) PCR-multiplexes for a genome wide framework of simple sequence repeat marker loci in cultivated sunflower. Theoretical and Applied Genetics 107: 6-19.

Tapia ME (1979) Historia y distribción geográfica. In: Tapia ME (ed.) Quinua y Kañiwa: Cultivos Andinos. Bogota: Instituto Interamericano de Ciencias Agrícolas, pp. 11-19.

Tapia ME, Mujica SA and Canahua A (1980) Orígen, distribución geográfica, y sistemas de producción en quinua. Primera Reunion Sobre Genética y Fitomejoramiento de la Quinua. Puno: Universidad Nacional Técnica del Altiplano, Instituto Boliviano de Technologia Agropecuaria, Instituto Interamericana de Ciencias Agricolas, Centro de Investicación Internacional para el Desarollo, pp. A1-A8.

Todd JJ and Vodkin LO (1996) Duplications that suppress and deletions that restore expression from a chalcone synthase multigene family. Plant Cell 8: 687-699.

Tommasini L, Batley J, Arnold GM, Cooke RJ, Donini P, Lee D, Law JR, Lowe C, Moule C, Trick M and Edwards KJ (2003) The development of multiplex simple sequence repeat (SSR) markers to complement distinctness, uniformity and stability testing of rape (Brassica napus L.) varieties. Theoretical and Applied Genetics 106: 1091-1101.

Ward SM (2000) Allotetraploid segregation for single-gene morphological characters in quinoa (Chenopodium quinoa Willd.). Euphytica 116: 11-16.

Wilson HD (1988a) Quinoa biosystematics I: domesticated populations. Economic Botany 42: 461-477.

Wilson HD (1988b) Quinoa biosystematics II: free living populations. Economic Botany 42: 478-494. 